\title{
Desarrollo de materiales de base Fe a partir de la síntesis de precursores por aleado mecánico
}

\author{
A. GONZÁLEZ, J.J. SUÑOL, LL. ESCODA, LL. ARTALEJO \\ GRMT, Dept. de Física, Universidad de Girona 17071, España
}

\begin{abstract}
Se han producido mediante síntesis mecánica aleaciones ricas en $\mathrm{Fe}$, con $\mathrm{Zr}$ o Nb y con un no metal, B obteniéndose materiales nanoestructurados con una incipiente fase amorfa (entorno 12\%). Tanto el $\mathrm{Zr}$ y el $\mathrm{Nb}$ como el B facilitan la reducción del tamaño de los nanocristales. Se han optimizado las condiciones de proceso, minimizando el tiempo de molturación. Además, el posterior tratamiento térmico, controlado mediante calorimetría diferencial, de las diferentes aleaciones permite obtener la energía de activación de los procesos de cristalización, con valores entre 280 y $410 \mathrm{~kJ} \mathrm{~mol}^{-1}$ asociados al crecimiento cristalino. El control del tamaño de los nanocristales se enmarca en el estudio de la cinética de las transformaciones fuera del equilibrio en las que la fase incipiente tiene composición diferente a la de la fase inicial.
\end{abstract}

Palabras clave: aleado mecánico, DSC, XRD, aleaciones metal -no metal.

Development of Fe based materials obtained by mechanical alloying

Fe rich alloys with $\mathrm{ZrNi}$ or $\mathrm{Nb}$ and with non metallic B were developed by means of mechanical alloying. The material is nanocrystalline with a minoritary amorphous phase (about 12\%). The reduction of the crystallite size is favoured by the addition of $\mathrm{Zr}, \mathrm{Nb}$ or $\mathrm{B}$. The mechanical alloying parameters were optimised to obtain a nanocrystalline state at low milling time. Furthermore, the apparent activation energy of the crystallisation processes was obtained by thermal treatment, controlled by differential scanning calorimetry. Typical values between 280 and $410 \mathrm{~kJ} \mathrm{~mol}^{-1}$ are associated to crystalline growth. The control of the crystal size is related to the kinetic study of the non-equilibrium transformations where the incipient phase as different composition that the initial phase.

Keywords: mechanical alloying, DSC, XRD, metal - non metal alloys

\section{INTRODUCCIÓN}

La síntesis de aleaciones mediante la técnica del aleado mecánico (MA) permite la obtención tanto de fases de equilibrio como de no equilibrio, incluyendo materiales nanoestructurados, amorfos, o la extensión de los límites de solubilidad (1-3). Durante el aleado mecánico, las partículas de polvo de los precursores son sometidas a sucesivas fracturas y soldaduras en frío originadas por fenómenos de percusión y de abrasión. Las superficies frescas resultantes tras la fractura facilitan la formación de nuevas partículas y la aleación entre los diferentes precursores en forma de capas con la correspondiente interdifusión.

Recientemente, aleaciones de base $\mathrm{Fe}$ (conteniendo nanocristales de $\alpha$-Fe de tamaño inferior a los 100nm) con algún elemento no metálico como el $\mathrm{B}$ del tipo Fe-Zr-B o Fe-Nb-B han sido obtenidas mediante nanocristalización inducida térmicamente (4) de aleaciones amorfas previamente producidas en forma decinta mediante técnicas de solidificación rápida (5-7). Estos materiales son objeto de estudio por sus propiedades magnéticas como la permeabilidad efectiva y la densidad de flujo de saturación magnético (8-10). Sus propiedades les hacen materiales alternativos en dispositivos magnéticos como transformadores de frecuencia, motores, generadores y sensores. El aleado mecánico es una vía alternativa para la obtención directa de nanocristales en el seno de una matriz amorfa (11). En el presente artículo se realiza la caracterización térmica, morfológica y estructural de dos aleaciones metal - no metal del tipo Fe-X-B ( $\mathrm{X}=$
$\mathrm{ZrNi}, \mathrm{Nb}$ ) obtenidas mediante aleado mecánico. El tiempo máximo de molturación, 40h, corresponde a la obtención de la nanoestructura con una amortización parcial.

\section{PARTE EXPERIMENTAL}

La síntesis mecánica se ha realizado en un micromolino planetario (Fritsch Pulverisette P7) a partir de elementos y compuestos de elevada pureza $(<99.0 \%)$ y con un tamaño de partícula pequeño $(10-100 \mu \mathrm{m})$. Las composiciones metal - no metal analizadas en este artículo son $\mathrm{Fe}_{74} \mathrm{Ni}_{14} \mathrm{Zr}_{6} \mathrm{~B}_{6}$ y $\mathrm{Fe}_{85} \mathrm{Nb}_{9} \mathrm{~B}_{6}$, que denominaremos A y $\mathrm{B}$ respectivamente. El aleado mecánico se ha producido en el interior de contenedores de acero inoxidable al $\mathrm{Cr}-\mathrm{Ni}$ conjuntamente con bolas del mismo material en una atmósfera inerte, Ar. La relación en peso entre las bolas y el polvo es 5:1 y la velocidad de rotación del dispositivo fue de 600 r.p.m. Se ha procedido a la extracción del material para ser analizado a las 10, 20 y 40 horas de proceso.

La caracterización térmica de las aleaciones se ha realizado en un calorímetro diferencial de barrido (DSC), en un equipo DSC30 de Mettler-Toledo, en atmósfera inerte, Ar. El análisis morfológico se ha realizado mediante microscopía electrónica de barrido (SEM), en un equipo DSM960A de Zeiss. El análisis composicional se ha realizado mediante microanálisis de dispersión de energías de rayos X (EDX) acoplado al 
microscopio y mediantre espectroscopía de plasma acoplado inductivamente (ICP) en un equipo Liberty-RL ICP Varian. La caracterización estructural mediante difracción de rayos $X(X R D)$ se ha realizado en un equipo D-500 Siemens con $\mathrm{Cu}$ $\mathrm{K}_{\alpha}$.

\section{RESULTADOS Y DISCUSIÓN}

La contaminación detectada mediante EDX e ICP aumenta con el tiempo de molturación. No obstante, los resultados de ICP muestran sólo una ligera contaminación (2.5 at.\% y 2.3 at.\% para las aleaciones A y B respectivamente) proveniente del medio de molienda ( $\mathrm{Fe}, \mathrm{Ni}$ y $\mathrm{Cr}$ ) tras 40 horas de proceso. Resultados similares se han encontrado en aleaciones de Fe-Si (12) y Fe-Nb-B (13). Los valores obtenidos mediante EDX son locales y básicamente superficiales y muestran una

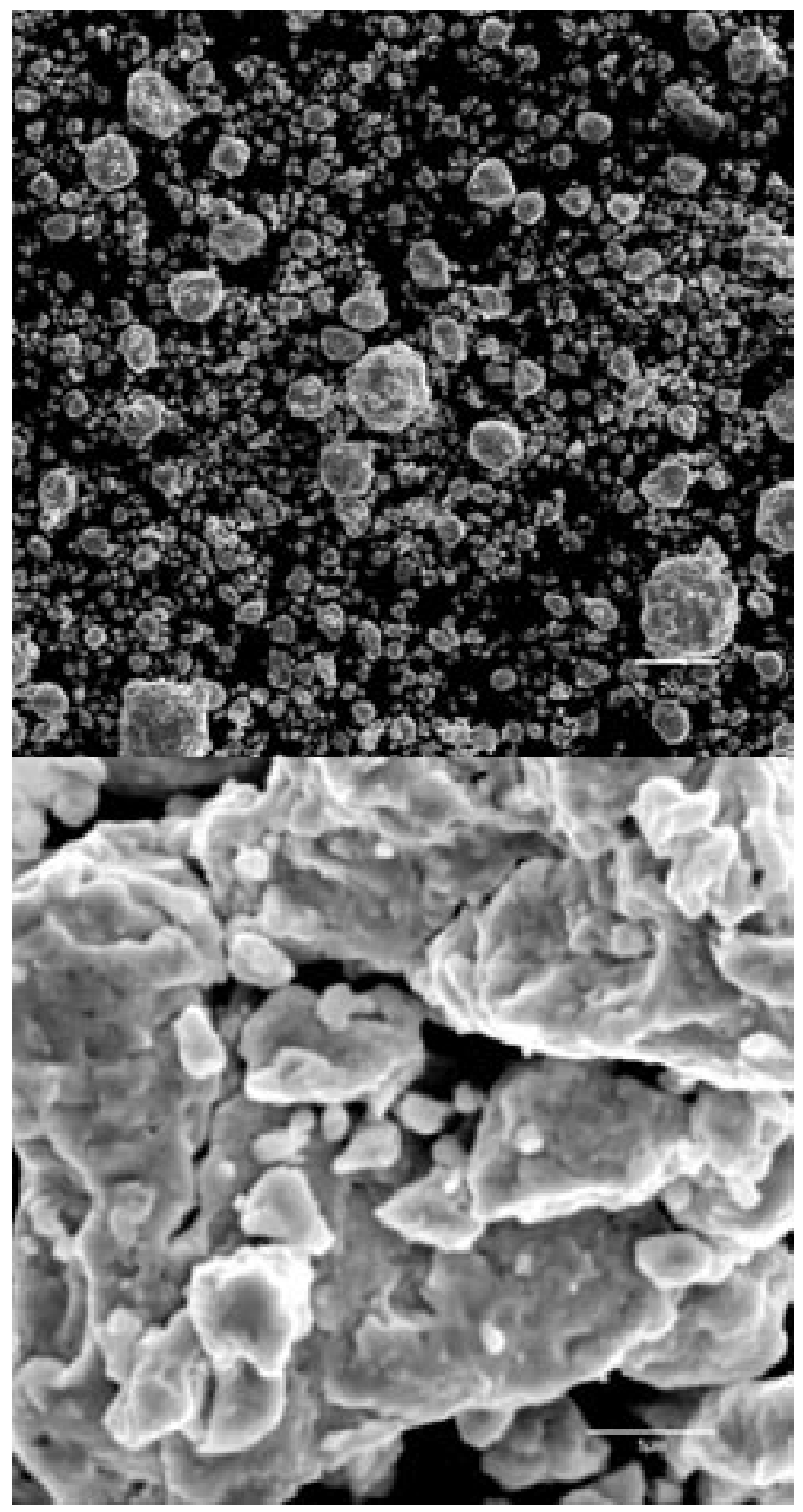

Fig. 1- Micrografías obtenidas mediante SEM de las aleaciones A (parte superior) y B (parte inferior) tras 40h de aleado mecánico. gran dispersión. En concreto, los valores detectados para el contenido de oxígeno tras $40 \mathrm{~h}$ de MA son $2.5 \pm 0.5$ at. $\%$ y 2.9 \pm 0.5 at.\% en las aleaciones A y B respectivamente. La figura 1 muestra la morfología de las aleaciones tras 40h de MA, en la muestra A una visión general y en la muestra $\mathrm{B}$ un detalle. Al aumentar el tiempo de MA, el tamaño de partícula promedio disminuye, alcanzando valores entorno $5 \mu \mathrm{m}$ tras $40 \mathrm{~h}$.

La formación de la estructura nanocristalina ha sido evaluada mediante difracción de rayos X. Las figuras 2 y 3 muestran los difractogramas correspondientes a las aleaciones A y B tras 0, 10, 20 y 40 de MA. El método de Rietveld permite constatar la presencia de una fase amorfa (entorno 12\%). El tamaño de los nanocristales es de $13 \pm 1 \mathrm{~nm}$. En la aleación B el valor obtenido corresponde a $10 \pm 1 \mathrm{~nm}$.. El parámetro de red aumenta con el tiempo de MA siendo mayor que el correspondiente a la fase $\alpha-\mathrm{Fe}, \mathrm{a}=0.28664 \mathrm{~nm}$, indicando la presencia de otros elementos, en concreto tras $40 \mathrm{~h}$ de MA el valor es $\mathrm{a}=0.2875(7)$ y $\mathrm{a}=0.2871(6)$ para las aleaciones A y B respectivamente. Análisis estructural complementario mediante espectroscopía Mössbauer de transmisión en aleaciones de composiciones similares confirman la formación de fases nanocristalinas sin una significativa formación de fase amorfa (13).

La estabilidad térmica de las aleaciones producidas se ha analizado mediante DSC. La estabilidad térmica es un factor importante en la determinación de la aplicabilidad de los materiales de estructura metaestable. En el caso del aleado mecánico, se producen fenómenos asociados a la relajación estructural del material tensionado mecánicamente, y la recristalización activada térmicamente.

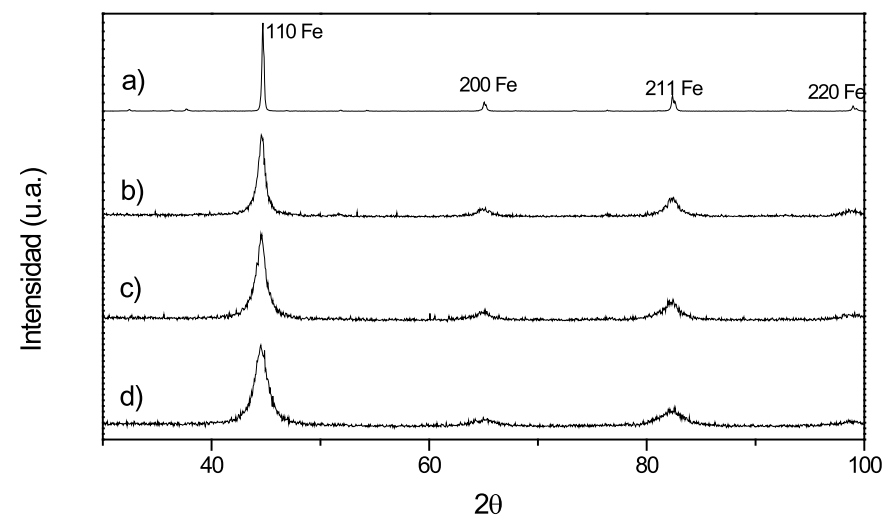

Fig. 2- Difractogramas de rayos $\mathrm{X}$ de la aleación $\mathrm{Fe}_{74} \mathrm{Ni}_{14} \mathrm{Zr}_{6} \mathrm{~B}_{6}$ tras: (a) $0 \mathrm{~h}$ de MA, (b) 10h de MA, (c) 20h de MA y (d) 40h de MA.

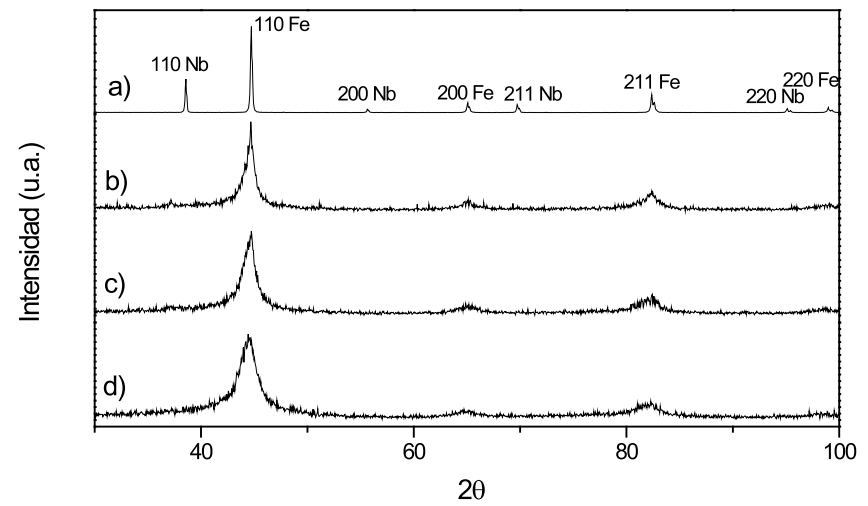

Fig. 3- Difractogramas de rayos $X$ de la aleación $\mathrm{Fe}_{85} \mathrm{Nb}_{9} \mathrm{~B}_{6}$ tras: (a) $0 \mathrm{~h}$ de MA, (b) 10h de MA, (c) 20h de MA y (d) 40h de MA. 
Las figuras 4 y 5 muestran las curvas de DSC correspondientes a las aleaciones A y B. Tras 10h de MA ya se visualizan diversos procesos exotérmicos. El suave y amplio proceso exotérmico que se inicia entorno $400 \mathrm{~K}$ corresponde a la relajación estructural de las aleaciones, básicamente energía de deformación almacenada durante la molturación, siendo un proceso característico de las aleaciones obtenidas mediante MA (14-15). La relajación tiene lugar durante un amplio intervalo de temperaturas y se solapa con diversos procesos exotérmicos en forma de pico correspondientes a la recristalización de las aleaciones, proceso con energías de activación bien definidas. En cambio, la relajación puede ser modelada asumiendo un amplio espectro de energías de activación del proceso de relajación (16). Los procesos de cristalización están asociados al crecimiento cristalino y reorganización de las fases cristalinas.

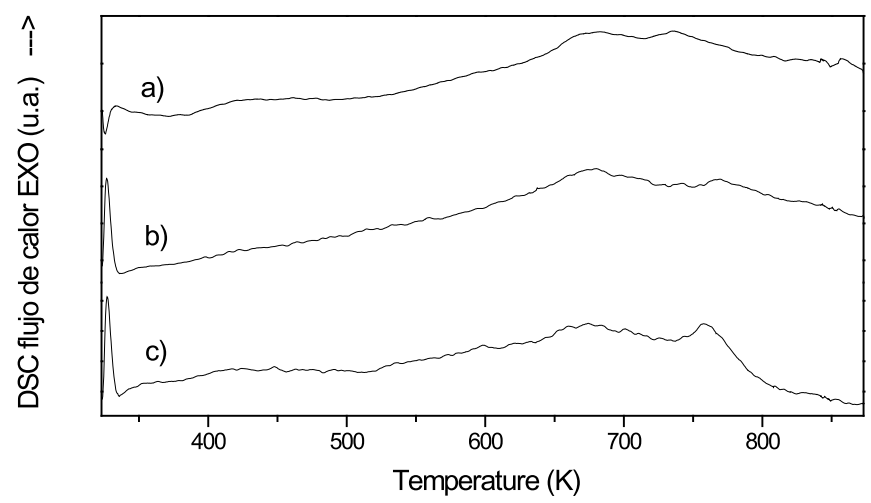

Fig. 4- Curvas calorimétricas de la aleación $\mathrm{Fe}_{74} \mathrm{Ni}_{14} \mathrm{Zr}_{6} \mathrm{~B}_{6}$ tras: (a) $10 \mathrm{~h}$ de MA, (b) 20h de MA y (c) 40h de MA.

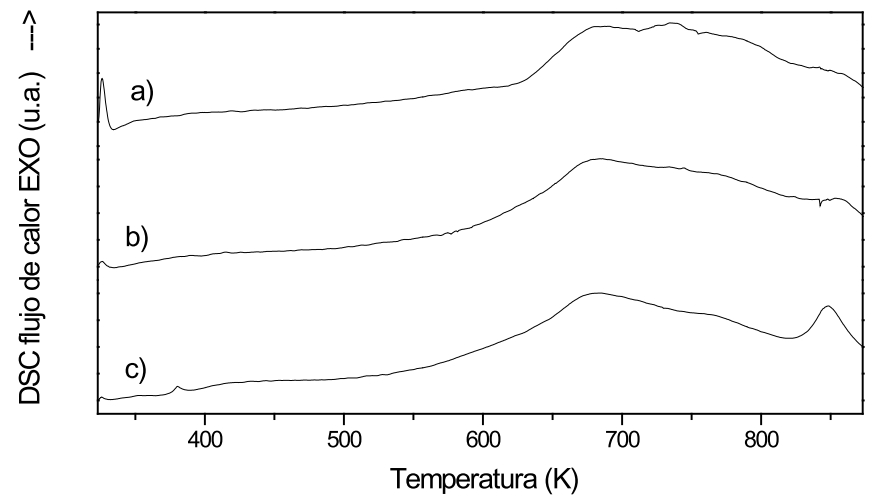

Fig. 5- Curvas calorimétricas de la aleación $\mathrm{Fe}_{85} \mathrm{Nb}_{9} \mathrm{~B}_{6}$ tras: (a) $10 \mathrm{~h}$ de MA, (b) 20h de MA y (c) 40h de MA.

En términos generales, la forma de las curvas calorimétricas tras 10, 20 o 40 horas de MA presentan un comportamiento similar. En todos los casos, el principal proceso de cristalización se activa térmicamente por encima de los $600 \mathrm{~K}$. En la aleación A, hay dos picos principales de cristalización, con una mayor estabilidad térmica el segundo al aumentar el tiempo de MA. La aparición de ambos procesos es típica de aleaciones obtenidas mediante aleado mecánico conteniendo Fe y Ni (17). En la aleación B tras 10h de MA aparecen diversos procesos exotérmicos muy solapados entre sí asociados a diferentes entornos de la fase $\alpha$-Fe. Al aumentar el tiempo de MA aparece entorno $850 \mathrm{~K}$ un proceso exotérmico que podría estar asociado a la formación de fases $\mathrm{Fe}-\mathrm{Nb}$ ya detectadas en aleaciones de composición similar (18-19). En la aleación B al aumentar el tiempo de MA el área asociada a los diversos procesos exotérmicos aumenta entorno un $50 \%$.

La energía aparente de activación, E, de los principales procesos de cristalización tras 40 de MA ha sido calculada empleando el método de Kissinger, asumiendo una relación lineal entre $\ln \left(\beta / \mathrm{T}_{\mathrm{p}}{ }^{2}\right)$ y $1 / \mathrm{T}_{\mathrm{p}}$ siendo $\beta$ la velocidad de calentamiento y $\mathrm{T}_{\mathrm{p}}{ }_{\mathrm{p}}$ la temperatura de pico (20). Se han obtenido los datos calorimétricos realizando calentamientos a diferentes velocidades: 2.5, 5, 10, 20 and $40 \mathrm{~K} \mathrm{~min}^{-1}$. Los valores obtenidos han sido $320 \pm 35 \mathrm{~kJ} \mathrm{~mol}^{-1}$ y $280 \pm 30 \mathrm{~kJ} \mathrm{~mol}^{-1}$ para la aleación A y $310 \pm 35 \mathrm{~kJ} \mathrm{~mol}^{-1}$ y $410 \pm 30 \mathrm{~kJ} \mathrm{~mol}^{-1}$ en la aleación B. Energías de activación con valores comprendidos entre 370 y $290 \mathrm{~kJ} \mathrm{~mol}^{-1}$ han sido encontrados en aleaciones del tipo Fe$\mathrm{X}-\mathrm{B}-(\mathrm{Cu}, \mathrm{Al}),(\mathrm{X}=\mathrm{Zr}, \mathrm{Nb}, \mathrm{ZrNb})$ (21-22), valores generalmente asociados a procesos de crecimiento cristalino (23).

Aleaciones de composición similar han sido obtenidas mediante solidificación rápida $(18,22)$. Los fenómenos de relajación estructural son usualmente menos energéticos (24). La fase amorfa obtenida cristaliza en dos etapas claramente diferenciadas correspondientes a la cristalización de la fase bcc $\alpha-\mathrm{Fe}$ y a la reordenación de esta con fases del tipo $\mathrm{Fe}_{2} \mathrm{X}$ y $\mathrm{Fe}_{3} \mathrm{X}(\mathrm{X}=$ no metal) respectivamente (18). Los procesos de cristalización se inician a temperaturas más elevadas, comportando una mayor estabilidad térmica de las fases de no equilibrio (24). En ocasiones, el segundo proceso de cristalización se inicia a temperaturas $>900 \mathrm{~K}$. Diseñando un tratamiento térmico adecuado sería posible controlar el tamaño de los nanocristales, análisis que se enmarca en el estudio de la cinética de las transformaciones fuera del equilibrio en las que la fase incipiente tiene composición diferente a la de la composición de la muestra. En las aleaciones A y B, el tratamiento térmico a $600^{\circ} \mathrm{C}$ produce un crecimiento de los nanocristales de la fase bcc Fe sin la formación de las fases del tipo FeX.

Los materiales obtenidos por MA están siendo sometidos a procesos de consolidación analizando los cambios estructurales y de comportamiento térmico. Desde el punto de vista tecnológico, se intenta desarrollar materiales con los que obtener piezas que supongan una mejora. En general, se busca un material con mayores prestaciones mecánicas que mantenga las características eléctricas y magnéticas de los materiales magnéticamente blandos.

\section{CONCLUSIONES}

Se han producido mediante aleado mecánico dos aleaciones (metal - no metal) de composición $\mathrm{Fe}_{74} \mathrm{Ni}_{14} \mathrm{Zr}_{6} \mathrm{~B}_{6}$ y $\mathrm{Fe}_{85} \mathrm{Nb}_{9} \mathrm{~B}_{6}$ en un estado nanocristalino con una incipiente fase amorfa (entorno 12\%) tras 40 horas de proceso. El tamaño de los nanocristales es de $13 \pm 1 \mathrm{~nm}$ en la aleación con $\mathrm{Zr}$ y de $10 \pm 1$ $\mathrm{nm}$ en la aleación con $\mathrm{Nb}$. Los valores de la energía aparente de activación son $320 \pm 35 \mathrm{~kJ} \mathrm{~mol}^{-1}$ y $280 \pm 30 \mathrm{~kJ} \mathrm{~mol}^{-1}$ en la aleación A y $310 \pm 35 \mathrm{~kJ} \mathrm{~mol}^{-1}$ y $410 \pm 30 \mathrm{~kJ} \mathrm{~mol}^{-1}$ en la aleación B. El estudio de la estabilidad térmica de las aleaciones permite la obtención de nanoestructuras de tamaño cristalino controlado. 


\section{AGRADECIMIENTOS}

Se agradece la financiación vía MICYT (MAT2003-08271C02-02) y DURSI (SGR2001-00190). A.G. agradece una beca predoctoral de la UdG.

\section{BIBLIOGRAFÍA}

1. C.C. Koch. 〈〈Synthesis of nanostructured materials by mechanical milling: Problems and opportunities $\rangle$. Nanostructured Mater. 9 13-22 (1997).

2. L.C.Zhang, J. Xu, E. Ma. 〈〈Mechanically alloyed amorphous Ti50(Cu0.45Ni0.55)(44-x)AlxSi4B2 alloys with supercooled liquid region $\rangle$. J. Mater. Res. 17 [7] 1743-1749 (2002).

3. K.A. Krivoroutchko, T. Kulik, V.I. Fadeeva, V.K. Portnoy. 〈〈Formation of stable and metastable phase in $\mathrm{Ni}-\mathrm{Al}-\mathrm{Nb}$ and $\mathrm{Ni}-\mathrm{Al}-\mathrm{Me}-\mathrm{C}(\mathrm{Me}=\mathrm{Ti}, \mathrm{Nb}$ or $\mathrm{V})$ powder systems during mechanical alloying and thermal treatment $\rangle\rangle$. J. Alloy Compd. 333, 225-231 (2002).

4. K. Suzuki, N. Kataoka, A. Inoue, A. Makino, T. Masumoto. 〈〈High saturation magnetization and soft magnetic properties of bcc Fe-Zr-B alloys with ultrafine grain-structure $\rangle$. Mater. Trans. JIM. 31 743-746 (1990).

5. A. Makino, T. Bitoh, A. Inoue, T. Masumoto. 〈(Nb-Poor Fe-Nb-B nanocrystalline soft magnetic alloys with small amount of $\mathrm{P}$ and $\mathrm{Cu}$ prepared by melt-spinning in air $\rangle$. Scripta materialia 48 [7] 869-874 (2003).

6. K. Suzuki, J.M. Cadogan. 〈(Effect of Fe-exchange-field penetration on the residual amorphous phase in nanocrystalline Fe92Zr8 $\rangle$. J. Appl. Phys., 87 [9] 7097-7099 (2000).

7. Y.Umakoshi, T. Nakano, T. Tsujimoto, W. Fujitani. 〈〈Microstructure and plastic deformation of melt spun amorphous $\mathrm{Fe}-\mathrm{Zr}-\mathrm{B}$ alloy ribbons containing nanocrystallites $\rangle\rangle$. Scripta Mater., 43, 349-354 (2000).

8. A. Kojima, S. Ito, A. Makino, A. Inoue. 〈 Soft magnetic properties of nanocrystalline $\mathrm{Fe}-\mathrm{Nb}-\mathrm{B}-\mathrm{P}$ alloys produced in the atmosphere by meltspinning method $\rangle$. Materials Transactions 42 [8] 1535-1539 (2001).

9. C.A.C. Souza, M.F. de Oliveira, J. E. May, W.J. Botta, N.A, Mariano, S.E. Kuri, C.S. Kiminami. 《(Corrosion resistance of amorphous and nanocrystalline FeM-B (M=Zr, Nb) alloys 〉. J. Non-Cryst. Solids 273 282-287 (2000).

10. J.S. Garitaonandia, P. Gorria, L. Fernández Barquín, J.M. Barandiarán. 〈〈Low-temperature magnetic properties of Fe nanograins in an amorphous Fe-Zr-B matrix $\rangle$. Physical Review B, 2 [9] 6150 (2000).
11. M.E. McHenry, M.A. Willard, D.E. Laughlin. 《〈Amorphous and nanocrystalline materials for applications as soft magnets $\rangle\rangle$. Prog Mater. Sci. 44291 (1999).

12. N. Malhouroux-Gaffet, E. Gaffet. 〈〈Solid-state reaction induced by post-

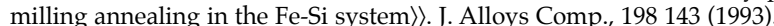

13. J.J. Suñol, A. González, J. Saurina, Ll. Escoda, T. Pradell, M.T. ClavagueraMora, N. Clavaguera. 〈〈Thermal and structural characterization of Fe$\mathrm{Nb}-\mathrm{B}$ alloys prepared by mechanical alloying $\rangle\rangle$. Materials Science and Engineering A 375 874-880 (2004)

14. S. Suriñach, M.T. Clavaguera-Mora, M.D. Baró, J. Segura, J.J. Suñol, N. Clavaguera. 〈(Preparation of Fe-Ni metal-metalloid amorphous powders by mechanical alloying $\rangle.$ Bol. Soc. Esp. Ceram. V., 31 369-374 (1992).

15. J.J. Suñol, T. Pradell, N. Clavaguera, M.T. Clavaguera-Mora. 〈〈Mossbauer spectroscopy study of the crystallisation behaviour of Fe-Ni-Si-P amorphous powders prepared by ball milling $\rangle\rangle$. J. Metast. Nanocryst. Mater., 10 525-530 (2001).

16. M.T. Clavaguera-Mora, J.J. Suñol, N. Clavaguera. 〈〈Relaxation kinetics of mechanically alloyed powders. Fe-Ni-Si-P: A case study $\rangle$. Mater. Sci. Forum 10 459-466 (2001).

17. J.J. Suñol, N. Clavaguera, M.T. Clavaguera-Mora. 〈 (Comparison of Fe-Ni-PSi alloys prepared by ball milling $\rangle$. J. Non-Cryst. Solids 287 114-119 (2001).

18. A. Makino, K. Suzuki, A. Inoue, T. Masumoto. 〈〈Magnetic properties and core losses of nanocrystalline Fe-M-B $(\mathrm{M}=\mathrm{Zr}$, Hf or $\mathrm{Nb})$ alloys $\rangle\rangle$. Mater. Sci. Eng. A 179127 (1994).

19. J.J. Suñol, T. Pradell, N. Clavaguera, M.T. Mora. 〈〈Ball milling of Fe40Ni40P20-xSix ( $x=6,10$ and 14): production and characterization $\rangle$. Philos. Magazine 83 2323-2342 (2003).

20. H. Kissinger. 〈〈Reaction kinetics in differential thermal analysis $\rangle\rangle$. Anal. Chem., 29 1702-1706 (1957).

21. P. Duhaj, I. Matko, P. Svec, J. Sitek, D. Janickovic. 〈〈Structural investigation of $\mathrm{Fe}(\mathrm{Cu}) \mathrm{ZrB}$ amorphous alloy $\rangle$. Mater. Sci. Eng B 39208 (1996).

22.A-H.Mansour,J.Barry. 〈(NanocrystallizationkineticsofFe85.5Zr4Nb4B5.5Al1 amorphous alloy $\rangle$. J. Mater. Sci. Letters 17 1125-1127 (1998).

23. J.J. Suñol, A. González, J. Saurina. 〈 $\mathrm{ZrNi}$ ) alloys prepared by mechanical alloying $\rangle\rangle$. J. Therm. Anal. Calorim. 72 329-335 (2003).

24. T. Pradell, J.J. Suñol, N. Clavaguera, M.T. Clavaguera-Mora. 〈〈Crystallization behaviour of Fe40Ni40SixP20-x $(x=6,10,14)$ amorphous alloys $\rangle$. J. NonCryst. Solids, 276113 (2000)

Recibido: 20.04.05

Aceptado: 17.06 .05

Trabajo presentado en el Congreso de Materiales Madrid 2002. 